\title{
A Note on the Effect of Haemorrhage upon the Blood Coagulation Time in Rabbits.
}

\author{
By \\ BUNKICHI KAMEI. \\ (象 井 文 莒) \\ (From the Physiological Laboratory of Prof. Y. Satake, \\ Tohoku Imperial University, Sendai.)
}

It seems generally accepted that haemorrhage calls forth an acceleration in the clotting of the blood. However the testimony in favour of such view is rather meager, that is, the reports are either fragmentary or loaded with discordant results. Entirely harmonizing data are to be seen in a report of A kai on rabbits. ${ }^{1)}$

In attacking the same problem on rabbits the present writer has come to witness a different feature which is seemingly worth while to present here.

\section{Methods :}

Rabbits of both sexes were used. Double splanchnicotomy was carried out by opening the abdominal cavity, or in a few cases by the lumbar route, about three weeks or more before the haemorrhage experiment. In the majority of cases the animal was tied on the table during the whole experiment, while in some cases the blood samples were taken from the rabbits, held in the hands of an assistant. No narcosis was resorted to at all.

The blood coagulation time was determined by means of Hira y a ma's modification ${ }^{2}$ of the graphic coagulometer of Cannon and Mendenhall. In the tapered glass tube, constructed according to Hir ay ama, a small modification was tried in order to make uniform the amount of blood which comes into contact with the copper wire and the glass wall. The distance

1) Aka i, Nippon Gekagakkai Zasshi, 1923, 24, 1323.

2) Hira yam a, Tohoku J. Exp. Med. 1925, 6, 160. 
from the two-centimeter-mark on the narrow portion to the broader one was made 5 mms. and marked there.

The blood samples for the coagulation time assay were taken from the femoral artery; when the collection was finished a small bull-dog clamp was laid and the artery outside of the clamp was carefully rinsed and rubbed with vaseline by means of the cotton attached to the end of a small rolling cotton holder, and covered with a piece of cotton immersed with 'Ty rode's fluid to avoid dryness.

The bleeding was done from the carotid artery as a rule. The magnitude of bleeding is below referred to in its ratio to the normal circulating blood quantity, the latter being taken as 5.44 per cent of the body weight. ${ }^{3)}$

\section{Experimental Data :}

About one hour was allowed to elapse after preparing the femoral and carotid arteries for taking the blood, with the purpose of avoiding a variation in the coagulation time, which possibly might occur by that kind of handling.") Then the blood samples were taken from time to time from the femoral artery, and the coagulation time was determined. Their average was taken as the initial value of the blood coagulation time of that individual rabbit. The values thus obtainable by the method of CannonMendenhall-Hiray am a were found for 50 normal, i. e. non-splanchnicotomized individuals as 3.8-7.1 minutes, mean 5.2 minutes. Two other rabbits showed an extraordinarily long coagulation time, as 31.4 and 16.0 minutes. Those in the doubly splanchnicotomized, 27 in number, were 3.7-6.6 minutes, on an average 5.0 minutes. In three other rabbits so operated on the blood clotted more slowly ; 8.9, 9.2 and 13.0 minutes were noted. The coagulation time in the normal rabbits of Hiray ama, obtained by one and the same method of determination, is unmistakably longer than ours.

Thrce individuals were fastened on the table, the femoral artery was laid bare and the blood specimens for measuring the coagulation time were drawn from time to time in the course of 4 or 5 hours. No special bleeding of a large amount was undertaken. The coagulation time was found nearly uniform during that spell, \pm 0.5 minute (the vertical rod was moved every 30 scconds) or \pm 10 per cent of the mean congulation time being found the maximum variation and no definite tendency being found in the fluctua-

8) W. J. Me ek and II. S. Gasser, Am. J. Physiol, 1918, 47, 302. 
tion curve. One tenth of the total blood volume was shed from two rabbits; the blood coagulation time underwent no visible deviation from the control experiments. The mean initial coagulation time was 5.8 and 6.7 minutes.

In 9 normal, i. e. non-splanchnicotomized and 10 splanchnicotomized the bleeding of one eighth of the total blood quantity was conducted; the time which was consumed in collecting that amount of blood from the carotid artery was noted as $9-34.5$ seconds, ncan 15 seconds for the former set of animals, and 6.8-47 seconds, mean 24 seconds for the latter. In the cases of normal aniwals the accelerating influence of the bleeding upon the coagulation time was detectable only in three cases. The coagulation time was shortened from 4.9, 5.6, 4.5 and 4.8 minutes to 4.0, 4.5, 3.5 and 3.0 minutes respectively. The shortest coagulation time appeared in ten to sixty minutes after bleeding. In three cases a prolongation instead of shortening took place, $6.1,6.6$ and 4.9 minutes being the mean initial clotting time and $9.5,8.5$ and 8.0 minutes the longest after bleeding, which made its appearance two hours thereafter or later. 'The mean initial coagulation time was 4.0 to 6.6 minutes for 9 normal animals, and 4.4 to 13.0 minutes in 10 doubly splanchnicotomized. In nearly all of the cases in the latter group of animals an augmentation resulted in the clotting by the haemorrhage. The figures are 4.5 minntes to 3.5 minutes, 4.9 to $3.5,9.2$ to 4.0 , 5.3 to $3.5,13.0$ to $7.0,6.2$ to $4.5,6.6$ to 4.5 and 8.9 to 6.0 . The smallest coagulation time was detected about ten minutes to two hours after bleeding. In the two remaining animals the variation was not large.

One fifth of the total blood quantity was lost in 14 non-splanchnicotomized and 15 splanchnicotomized animals. The bleeding consumed 8 to 81 seconds for the former series of animals, with two exceptionally slow ones, as 119 and 267 seconds, and 22 to 85.5 seconds for the latter, also with some exceptional cases $(117,162$ \& 186 seconds). In general the velocity with which the blood was shed was smaller in the doubly splanchuicotomized ones. An augmenting effect of haemorrhage upon the clotting was rearly detectable in the normal animals when one fifth of the total blood was shed. In the majority of cases the bloods taken after bleeding clotted faster. It is worthy of note that the shortening was remarkable in the cases where the initial clotting time was extraordinarily long, as 31.4 or 16.6 minutes. The time point when the shortest coagulation time was recorded did not differ from the cases of bleeding of one eighth. This time the double splanchnicotomy did not modify the results so much as the above quoted cases or rather the accelerating effect of hacmorrhage upon the 
coagulation time was made obscure. It must be remarked here that in Rabbit No. 70 the second haemorrhage of ca. 7 c.c. resulted in a great acceleration in the clotting as $\mathbf{1 . 5}$ minutes in contrast to 9 minutes in a short interval. In Rabbits No. $72 \& 74$ the bleeding was tried on the second day of fasting.

The blood quantity corresponding to one third of the total quantity was drawn from 23 non-splanchnicotomized and 5 doubly splanchnicotomized rabbits. In the former group 24-300 seconds, in the majority of cases 24-66 seconds, were required for collecting. In the latter group it was 80-162 seconds. The accelerating influence of the haemorrhage upon the coagulation time was in general recorded, and the shortening was excessive in some cases. Excessive shortening was manifest within a half hour after the bleeding. But on a closer examination there were some instances where the shortening of the clotting was not scen at all or only with some difficulty, and in such cases the time required for collecting the blood was relatively short. In all of the doubly splanchnicotomized rabbits the haemorrhage invariably hastened the clotting.

The tables loaded with the data are given at the end of this paper.

\section{Discussion :}

The view seemingly generally accepted that bleeding hastens the coagulation of blood in rivo holds true also in the present investigations on the whole, and the tendency that the magnitude of the hastening depends on the amount bled is not to be denied, roughly speaking. However, the ontcome of this kind of research eeems not to be so uniform, but rather inconsistent, contrary to the blood sugar study. ${ }^{4}$ The experimental conditions in the present investigations, I believe, were by no means complicate, but the outcome was not so consistent. So also were the results of our investigations on dogs, which were done in succesion to the present. ${ }^{5}$ The velocity with which epinephrine is liberated from the suprarenal gland, the blood sugar level and the blood coagulation time were sinultaneously determined on non-fastened, non-narcotized dogs, before and after bleeding. The coagulation time determination failed to yield so fairly uniform features contrary to the two other estimations.

One eighth of the total blood quantity often failed to accelerate the coagulation in normal rabbits; this seems therefore to be assumable as the

4) Tach i, Tohoku J. Exp. Med., 1928, 10, 96.

5) Saito, Kamei and Tachi, Ibid., 1928, 11, 205. 
smallest amount of bleeding sufficient to occasion the faster clotting. The double splanchnicotomy modified this figure definitely, that is, the bleeding of one eighth of the total blood amount acted to augment the clotting in the majority of cases, a fact of worthy of note.

The influence of the splanchnicotomy apparently contradicts the previous communications as follows: Gray and $\mathrm{Lun} \mathrm{t}^{(\boldsymbol{\omega})}$ were able to detect that haemorrhage fails to decrease clotting time after exclusion of the abdominal circulation, in accordance with the findings of some old physiologists rê the influence of the exclusion of the abdominal circulation upon the coagulation time. Adrenaline modifies the clotting time in viro, ${ }^{7}$ ) and stimulation of the splanchnic nerve calls forth the hastening of the cougulation, when the suprarenal gland is not interfered with ${ }^{\mathrm{S})}$ etc. Only the report of $\mathrm{Ba}$ rlow and Ell is apparently does not disagree with the present findings. The double suprarenalectomy in cats was followed by the diminution of the congulation time of blood. ${ }^{9)}$

\section{Summary:}

The coagulation time was determined for the bloods from the fastened rabbits before and after bleeding by means of Hir a y a ma's modification of the graphic coagulometer of Cannon and Mendenhall.

The accelerating action of bleeding upon the clotting was infrequently detectable in normal rabbits when one eighth of the total blood amount was shed. The double splanchnicotomy was found to facilitate the manifestation of this action in the cases of bleeding of a similar amount of blood.

With the magnitude of bleeding the acceleration in the blood coagulation increased in the consistency of occurrence and in the severity, roughly speaking. However this rule can not be applied to the coagulation time study with such certainty as in the case of the hyperglycaemia due to bleeding.

Further in the instances of one third of the total blood amount, a certain relation was perceived to exist, though not so decidedly, between the velocity with which the blood flowed out and the shortening of clotting.

6) H. Gray and L. K. Lunt, Am. J. Physiol., 1914, 34, 342 ff.

7) W. B. Cannon and H. Gray, Am. J. Physiol., 1914, 34, 232; Hirayama, Tohoku J. Exp. Med., 1925, 6, 160; J. La Barre, Arch. intern. Physiol., 1925, 25, 265.

8) W. B. Cannon and W. L. Mendeuhall, Ar. J. Physiol., 1914, 34, 243.

9) O. W. Barlow and M. M. Ellis, Am. J. Physiol., 1924, 70, 58. 

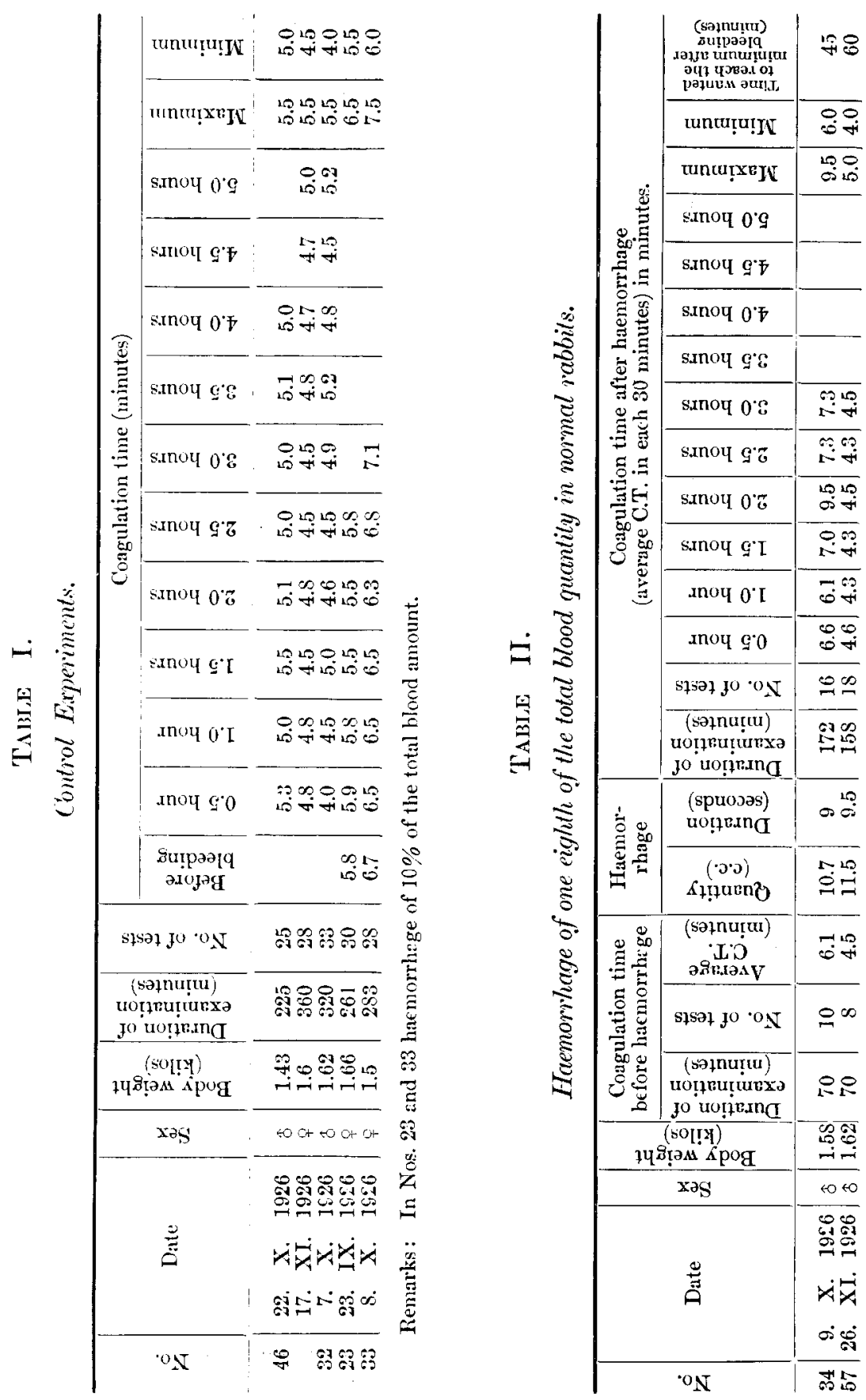


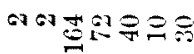

0001012010

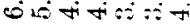
29 40, 4040

15

29025

यें

054

$190 . \div 0 \%$ 0 is is is 50010002015 ड $10^{\circ} 10^{\circ}+\operatorname{ti}^{\circ}$ $00002 \pi 4000$ O $\cos 50000 \div$ 15 20 सं 2005025 $00 \infty=\infty \omega$ 人1

काe $00001-7$

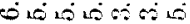
c) $610101-900$ S1010.8

6.

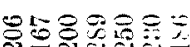

$\infty x+\infty=0$

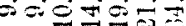

- $100000=$ or $0=0=$ is

$000: 150=$ $510+5-7$

$\infty 1-x+\infty=0$

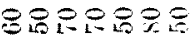

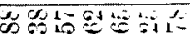

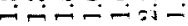

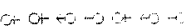

우운

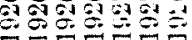

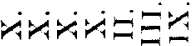

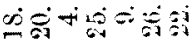

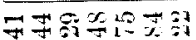

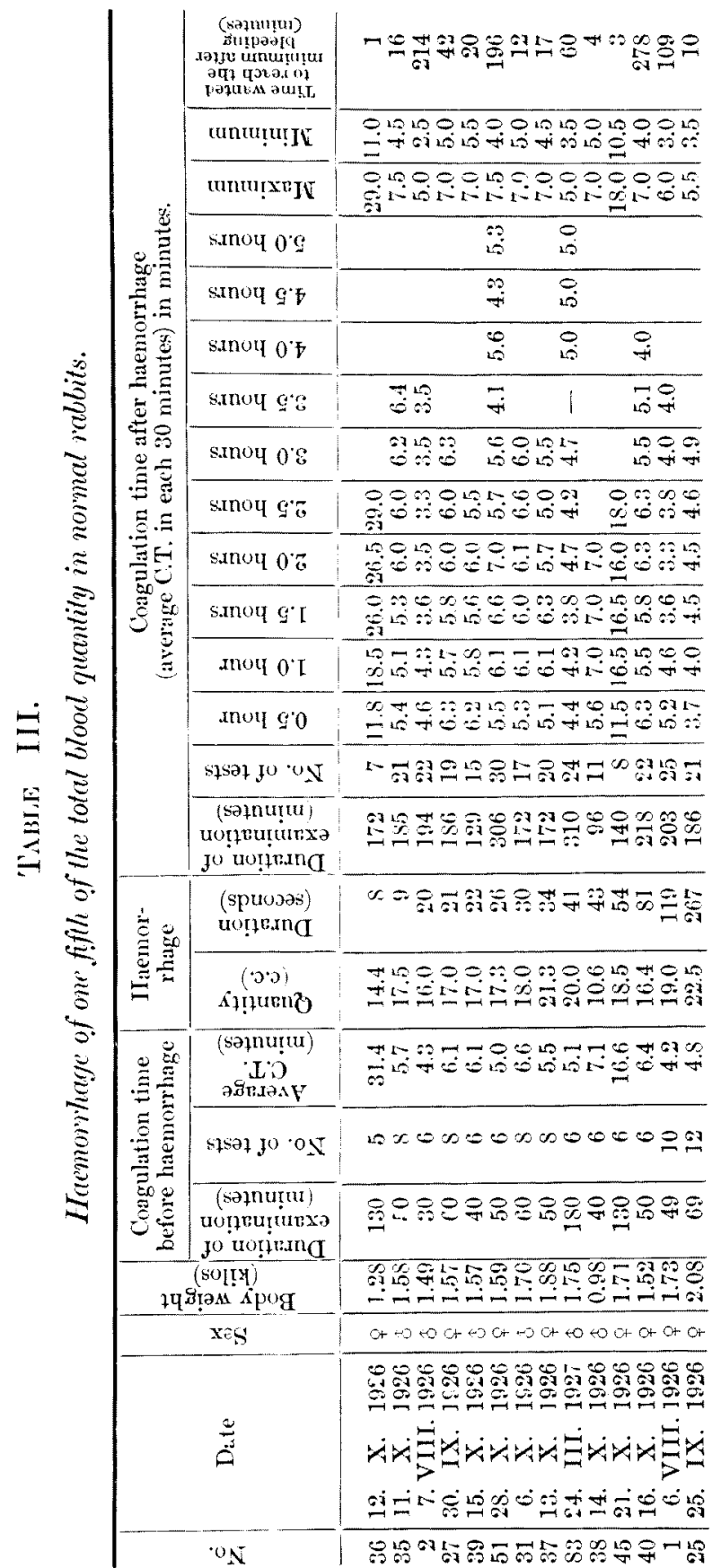




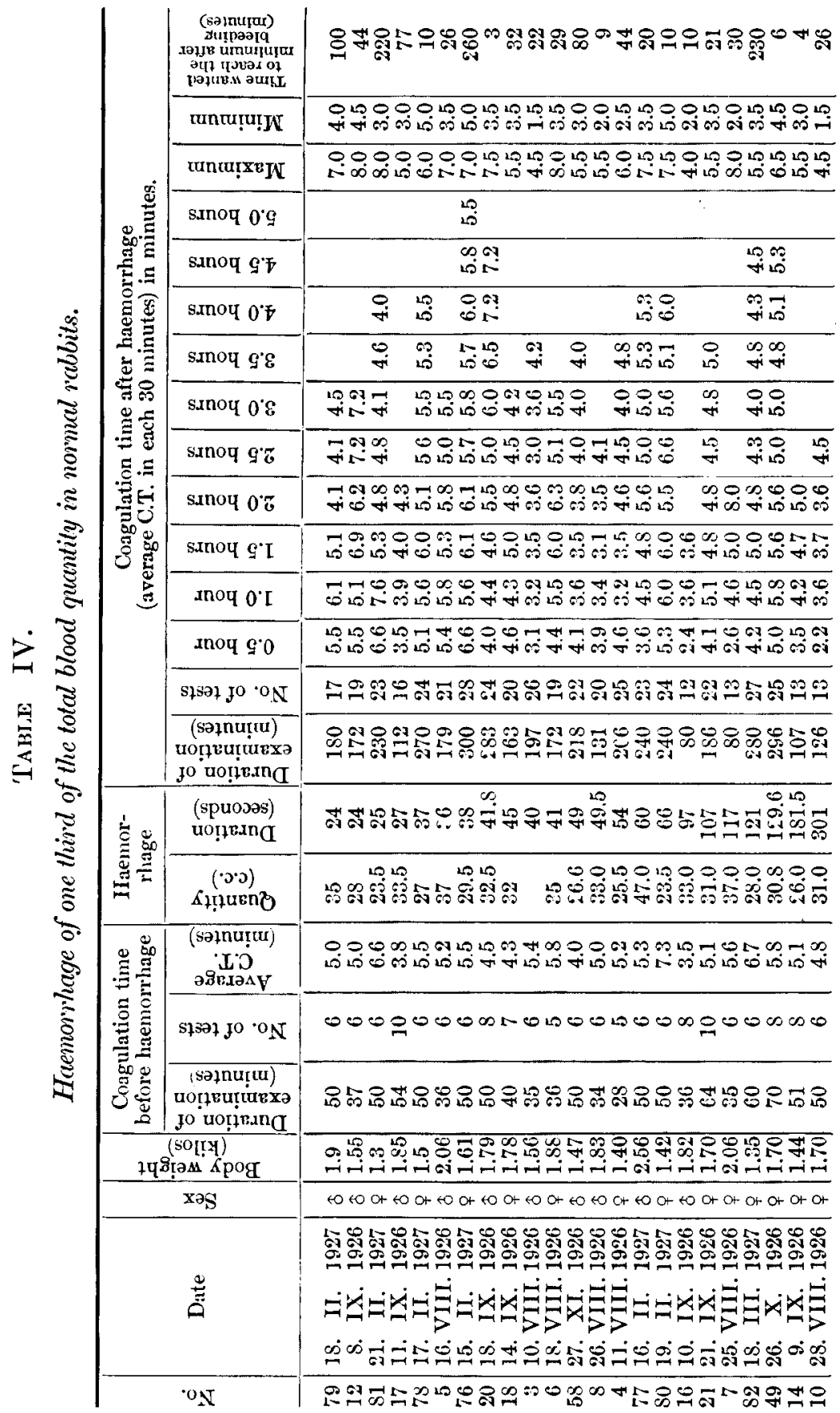




\begin{tabular}{|c|c|c|c|}
\hline \multirow{5}{*}{$\sum_{i}^{\infty}$} & \multirow{15}{*}{ 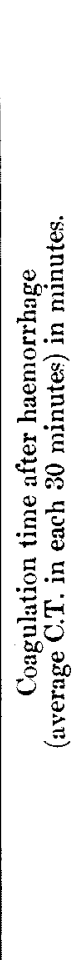 } & 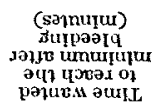 & 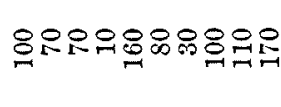 \\
\hline & & uncu!̣!IN & 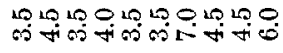 \\
\hline & & tundu!xeJ & 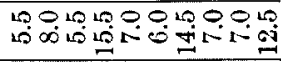 \\
\hline & & sinot $0^{\circ} 9$ & \\
\hline & & sunoy g' & $\operatorname{tin}$ \\
\hline \multirow{5}{*}{ 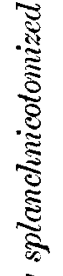 } & & sanoy $0^{\circ} \mathrm{F}$ & $\begin{array}{ll}\infty \\
+\infty\end{array}$ \\
\hline & & sunoy 98 & 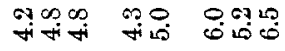 \\
\hline & & sinot $0^{\circ} 8$ & 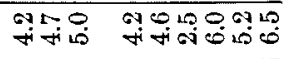 \\
\hline & & sinot $9 \%$ & L \\
\hline & & Sanoy $0^{\circ}$ & 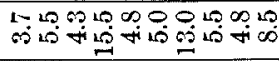 \\
\hline 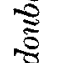 & & sinoy $g^{\prime} I$ & 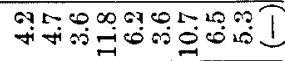 \\
\hline.$\approx$ & & Anot $0^{\circ} \mathrm{I}$ & न \\
\hline$\underset{\approx}{\approx}$ & & moy $9^{\circ} 0$ & 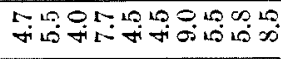 \\
\hline$\stackrel{\Xi}{\Xi}$ & & $\operatorname{słs} 37$ go $O N$ & 90905609890 \\
\hline$\frac{2}{8}$ & & 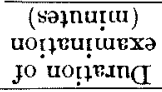 & 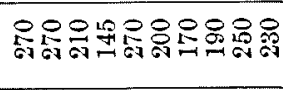 \\
\hline$\stackrel{\Xi}{E}$ & \multirow{2}{*}{ 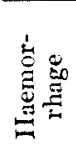 } & $\begin{array}{l}\text { (spuojas) } \\
\text { uogfing }\end{array}$ & 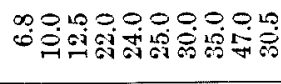 \\
\hline$\frac{\pi}{6}$ & & $\begin{array}{c}(\cdot \circ \cdot \partial) \\
\Lambda_{+!+u n t o}\end{array}$ & 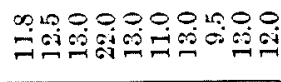 \\
\hline 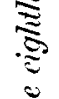 & $\stackrel{\stackrel{\oplus}{\rightleftarrows} \underset{\Xi}{\Xi}}{\Xi}$ & 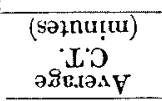 & 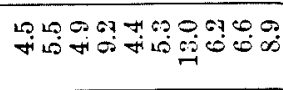 \\
\hline$\frac{5}{0}$ & 梽 & słsat Jo ON & $000000: 2000$ \\
\hline$\underset{8}{2}$ & 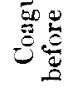 & 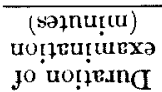 &  \\
\hline$\tilde{\xi}$ & & 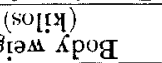 &  \\
\hline 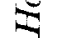 & & xas & to to to to to to to ot of ot \\
\hline & & 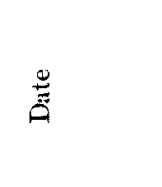 & 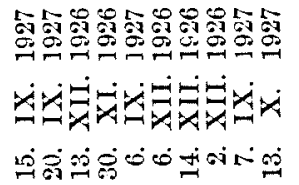 \\
\hline & & $0 \mathrm{~N}$ & \% \\
\hline
\end{tabular}




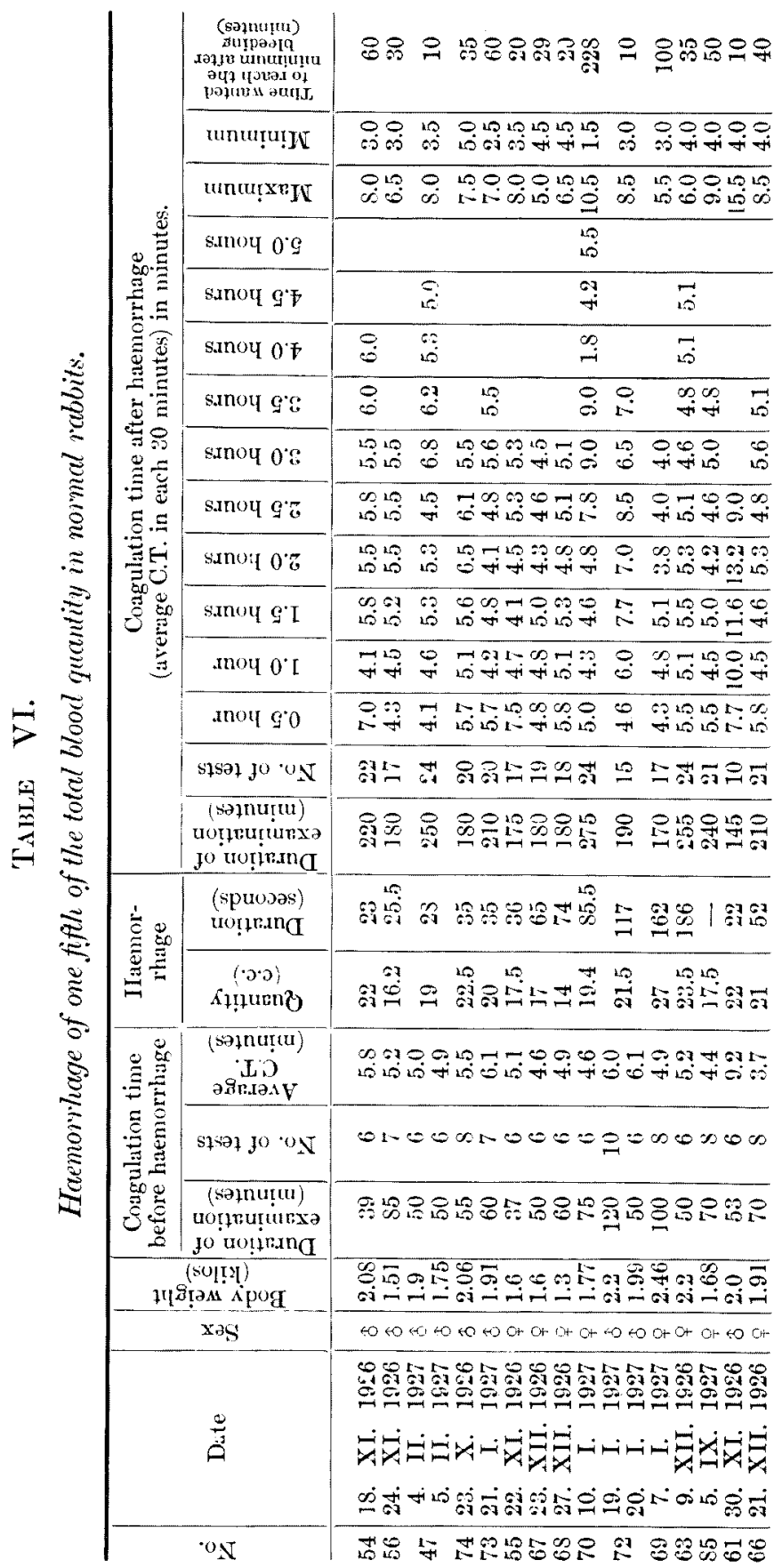




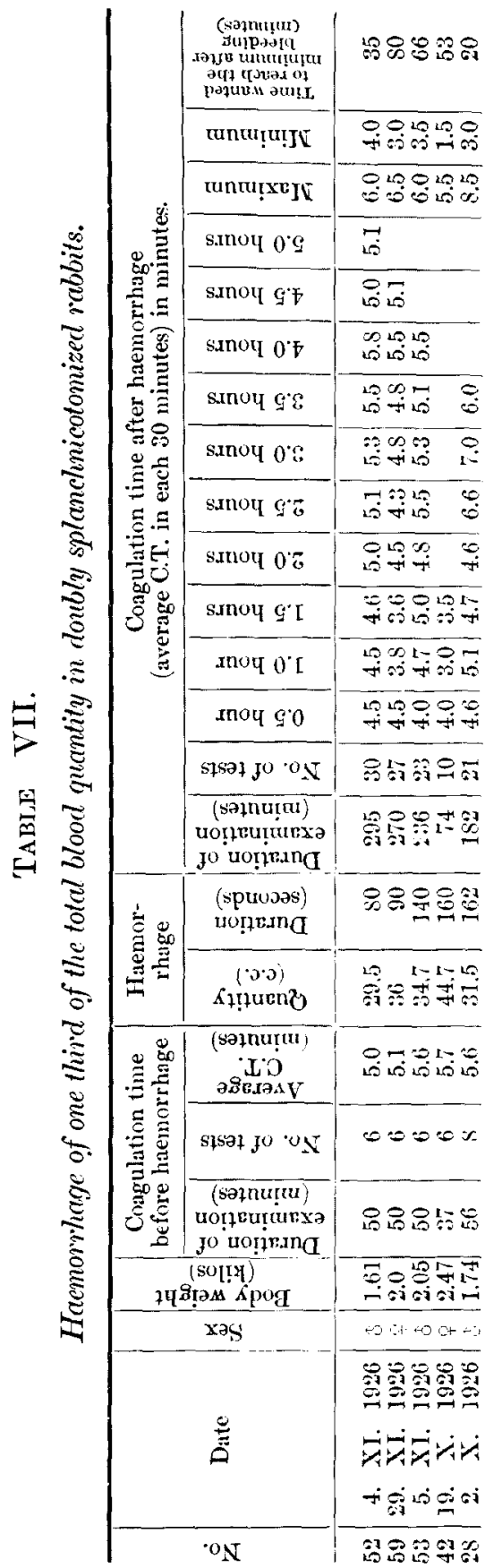

